Stud. Univ. Babeş-Bolyai Math. 64(2019), No. 2, 151-160

DOI: 10.24193/subbmath.2019.2.02

\title{
On a stochastic arc furnace model
}

\author{
Hans-Jörg Starkloff, Markus Dietz and Ganna Chekhanova
}

\begin{abstract}
One of the approaches in modeling of electric arc furnace is based on the power balance equation and results in a nonlinear ordinary differential equation. In reality it can be observed that the graph of the arc voltage varies randomly in time, in fact it oscillate with a random time-varying amplitude and a slight shiver. To get a more realistic model, at least one of the model parameters should be modeled as a stochastic process, which leads to a random differential equation.

We propose a stochastic model using the stationary Ornstein-Uhlenbeck process for modeling stochastic influences. Results, gained by applying Monte Carlo method and polynomial chaos expansion, are given here.
\end{abstract}

Mathematics Subject Classification (2010): 34F05.

Keywords: Electric arc furnace, random differential equation, Ornstein-Uhlenbeck process, Monte Carlo method, polynomial chaos expansion.

\section{Introduction}

An electric arc furnace (EAF) is used for melting metals in steel industry. One deterministic model of the EAF energy system is based on the instantaneous power balance of the system, which results in the following nonlinear ordinary differential equation

$$
k_{1} r^{n}(t)+k_{2} r(t) \frac{\mathrm{d} r(t)}{\mathrm{d} t}=\frac{k_{3}}{r^{m+2}(t)} i^{2}(t), \quad t \in \mathcal{I} .
$$

This equation describes how the arc radius $r$ depends on the arc current $i$, both are functions on a given time interval $\mathcal{I}$ (cf. [1]). The model coefficients $k_{1}, k_{2}$ and $k_{3}$ are positive. The parameters $m$ and $n$ belong to the set $\{0,1,2\}$ and reflect different working conditions of the arc furnace (cf. [4] or [5]). Equation (1.1) suggests, that the arc radius function $r$ is positive and should be prevented from getting zero. In case

This paper has been presented at the fourth edition of the International Conference on Numerical Analysis and Approximation Theory (NAAT 2018), Cluj-Napoca, Romania, September 6-9, 2018. 
the arc radius takes on the value zero one has to deal with a differential equation with singularities which requires additional investigations.

Between the arc voltage $u$, the arc radius $r$ and the arc current $i$ it holds the relationship

$$
u(t)=\frac{k_{3}}{r^{m+2}(t)} i(t), \quad t \in \mathcal{I} .
$$

In reality it can be observed that the arc voltage varies randomly in time, it oscillates with a random time-varying amplitude and the graph of the function shows a slight shivering (cf. [4] or [6]). To take this into account, it is better to model the arc voltage as a stochastic process. Here it is proposed to model the coefficient $k_{3}$ of equation (1.1) as a stochastic process and then solve the corresponding random differential equation.

Section 3 presents the model in more detail and gives some first results gained by the Monte Carlo method. Section 4 describes how polynomial chaos expansions can be applied.

The stochastic model is based on a deterministic one, for which for certain parameters explicit solutions of (1.1) can be given. This deterministic model was investigated for example in [6] and is recapped here in section 2.

\section{A deterministic model}

In this section assume the time interval is $\mathcal{I}=\mathbb{R}$. In this paper we want to consider the case $n=2$, for which (1.1) can be solved explicitly. For this parameter $n$ the equation (1.1) with the substitution $y=r^{m+4}$ results in the following linear ordinary differential equation

$$
\frac{\mathrm{d} y(t)}{\mathrm{d} t}=-\beta y(t)+f(t), \quad t \in \mathcal{I}
$$

with $f(t):=\frac{(m+4) k_{3}}{k_{2}} i^{2}(t)$ and $\beta:=\frac{(m+4) k_{1}}{k_{2}}>0$.

If we assume, that the arc current $i$ is a continuous function and that the initial value condition

$$
y\left(t_{0}\right)=y_{0}>0
$$

holds $\left(t_{0} \in \mathbb{R}\right)$, then $(2.1)$ has the unique continuously differentiable positive solution

$$
y(t)=y\left(t ; t_{0}, y_{0}\right)=y_{0} e^{-\beta\left(t-t_{0}\right)}+\int_{t_{0}}^{t} f(s) e^{-\beta(t-s)} \mathrm{d} s, \quad t \in \mathcal{I} .
$$

If we additionally assume, that $i$ is a $p$-periodic (with the period $p$ ), it is bounded and there exists a unique $p$-periodic solution of the differential equation (2.1). We get a formula of this $p$-periodic solution $y_{p e r}$ also by applying the pullback limit of $t_{0}$ on the initial value solution (2.3). It holds

$$
y_{\text {per }}(t)=\lim _{t_{0} \rightarrow-\infty} y\left(t ; t_{0}, y_{0}\right)=\int_{0}^{\infty} e^{-\beta s} f(t-s) \mathrm{d} s, \quad t \in \mathcal{I} .
$$

In [6] this periodic solution is referred to as a steady state solution of the system. Sometimes it is also called the equilibrium solution. 
According to the real world data the graph of the arc current has a sinusoidal shape. To satisfy this behavior the arc current function is chosen as

$$
i(t)=a \sin (\omega t), \quad t \in \mathcal{I}
$$

Here $a$ (amplitude) and $\omega$ (corresponding frequency) are positive parameters. If we apply (2.5), from (2.4) an explicit formula for the periodic solution can be derived.

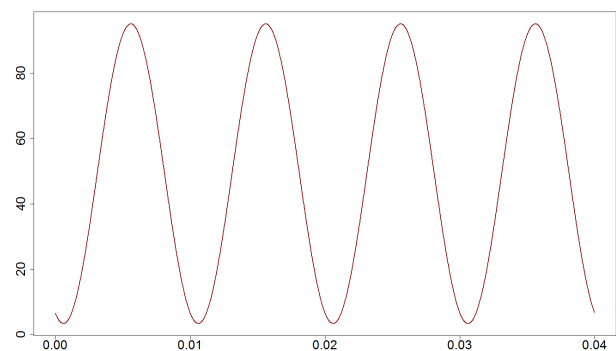

(a) periodic solution $y_{\text {per }}$

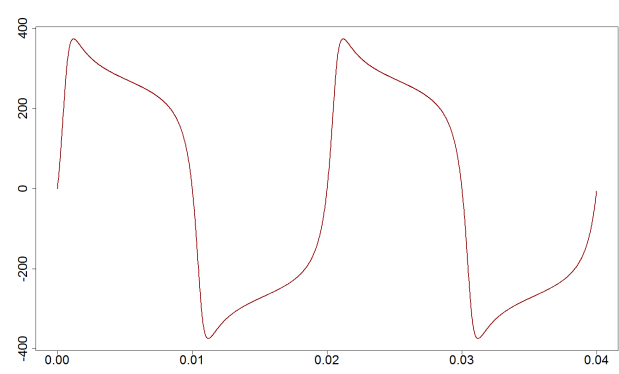

(c) arc voltage $u_{p e r}$

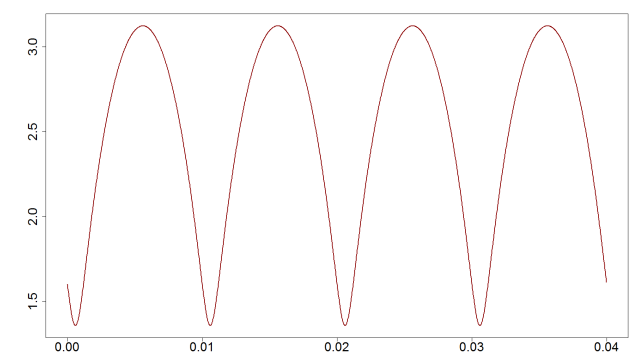

(b) arc radius $r_{p e r}=\left(y_{p e r}\right)^{\frac{1}{m+4}}$

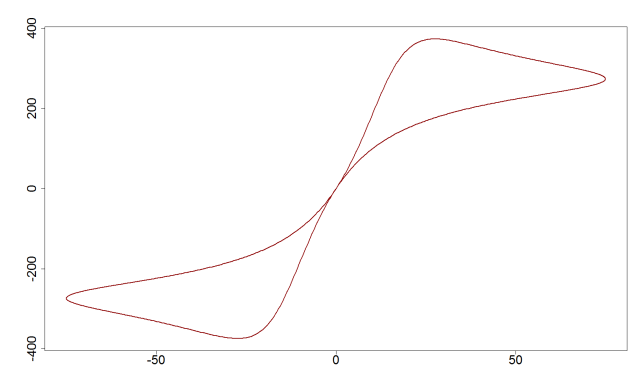

(d) voltage-current characteristic

FiguRE 1. Graphs of characteristics of the deterministic model with the following parameters

\begin{tabular}{cccccc}
\hline$a$ & $\omega$ & $m$ & $k_{1}$ & $k_{2}$ & $k_{3}$ \\
\hline 75 & $100 \pi$ & 0 & 2000 & 5 & 35 \\
\hline
\end{tabular}

Then also an explicit formula for the associated voltage function can be calculated. It holds

$$
y_{\text {per }}(t)=b[1-c \sin (2 \omega t+\psi)], \quad t \in \mathcal{I}
$$

and

$$
u_{p e r}(t)=d[1-c \sin (2 \omega t+\psi)]^{-\frac{m+2}{m+4}} \sin (\omega t), \quad t \in \mathcal{I}
$$


with the constants

$$
\begin{array}{rlrl}
b & =\frac{k_{3} a^{2}}{2 k_{1}}, & c & =\frac{1}{\sqrt{1+\left(\frac{2 \omega k_{2}}{(m+4) k_{1}}\right)^{2}},} \\
d=2^{\frac{m+2}{m+4}} k_{1}^{\frac{m+2}{m+4}} k_{3}^{\frac{2}{m+4}} a^{-\frac{m}{m+4}}, & \psi & =\arctan \left(\frac{(m+4) k_{1}}{2 \omega k_{2}}\right),
\end{array}
$$

depending on the model parameters $m, k_{1}, k_{2}, k_{3}, a$ and $\omega$.

Figure 1 shows graphs of the periodic solution $y_{\text {per }}$, the arc voltage $u_{p e r}$, the arc radius $r_{p e r}=\left(y_{p e r}\right)^{\frac{1}{m+4}}$ and the voltage-current characteristic, i.e., of the curve $\left(\left(i(t), u_{\text {per }}(t)\right): t \in \mathcal{I}\right)$.

\section{A stochastic model}

Here we want to propose a stochastic model, in which the coefficient $k_{3}$ from the former deterministic equation (1.1) is now a stochastic process. One of the modeling challenges is to make sure, that the inhomogeneity in equation (2.1) stays positive, which provides that the solution $y$ is always strictly positive and prevents the arc radius $r$ from getting negative. We take this into account by considering a stochastic process $\left(X_{t}\right)_{t \in \mathcal{I}}$ and multiplying $k_{3}$ with the non-negative stochastic process $\left(\left(1+X_{t}\right)^{2}\right)_{t \in \mathcal{I}}$. By inserting the stochastic process

$$
\left(k_{3}\left(1+X_{t}\right)^{2}\right)_{t \in \mathcal{I}}
$$

into equation (2.1) instead of the deterministic coefficient $k_{3}$, the differential equation (2.1) turns into the random ordinary differential equation

$$
\frac{\mathrm{d} Y_{t}}{\mathrm{~d} t}=-\beta Y_{t}+F_{t}, \quad t \in \mathcal{I}
$$

with $F_{t}=f(t)\left(1+X_{t}\right)^{2} .\left(Y_{t}\right)_{t \in \mathcal{I}}$ and $\left(F_{t}\right)_{t \in \mathcal{I}}$ are now stochastic processes.

Let $\mathcal{I}=\mathbb{R}$ be the considered time interval and $\left(X_{t}\right)_{t \in \mathcal{I}}$ be a stochastic process with continuous paths. If we assume additionally that holds $Y_{t_{0}}=y_{0}$ with a deterministic initial value $y_{0}>0$ and a deterministic initial time $t_{0} \in \mathbb{R}$, equation (3.2) has the unique pathwise random solution

$$
Y_{t}=y_{0} e^{-\beta\left(t-t_{0}\right)}+\int_{t_{0}}^{t} e^{-\beta(t-s)} F_{s} \mathrm{~d} s, \quad t \in \mathcal{I} .
$$

As a stochastic process $\left(X_{t}\right)_{t \in \mathbb{R}}$ we choose the stationary Ornstein-Uhlenbeck process (OUP). This is a Gaussian process with mean function constant zero and autocovariance function $\operatorname{Cov}\left(X_{t}, X_{t+h}\right)=\frac{\sigma^{2}}{2 \theta} \exp (-\theta|h|) \cdot \sigma$ and $\theta$ are positive parameters. If the time interval is restricted to $\mathcal{I}=[0, \infty)$, the OUP can be also considered as a solution of the stochastic differential equation

$$
\mathrm{d} X_{t}=-\theta X_{t} \mathrm{~d} t+\sigma \mathrm{d} W_{t}
$$


with a standard Wiener process $W=\left(W_{t}\right)_{t \geq 0}$ and an initial value $X_{0}$, which is independent of $W$ and follows a centred normal distribution with variance $\frac{\sigma^{2}}{2 \theta}$. This also allows to consider the equations (3.3) and (3.4) as a coupled system of stochastic differential equations

$$
\mathrm{d}\left(\begin{array}{c}
Y_{t} \\
X_{t}
\end{array}\right)=\left(\begin{array}{c}
-\beta Y_{t}+f(t)\left(1+X_{t}\right)^{2} \\
-\theta X_{t}
\end{array}\right) \mathrm{d} t+\left(\begin{array}{c}
0 \\
\sigma
\end{array}\right) \mathrm{d} W_{t} .
$$

Such coupled systems are investigated in many ways and special methods are developed for them, but this path is not followed here. Instead the random differential equation (3.2) is investigated.

With the choice of $X_{t}$ as Ornstein-Uhlenbeck process, there are explicit representations for certain characteristics of the solution (3.3) available. For example it holds for the mean function

$$
\mathrm{E}\left[Y_{t}\right]=y_{0} e^{-\beta\left(t-t_{0}\right)}+\left(1+\frac{\sigma^{2}}{2 \theta}\right) \cdot \int_{t_{0}}^{t} e^{-\beta(t-s)} f(s) \mathrm{d} s, \quad t \in \mathcal{I} .
$$

More challenging is the question of how to determine characteristics for the arc radius function $R$ and the arc voltage function $U$. The reason lies in the nonlinear relationship between the functions $R$ and $Y$, and respectively $U, Y$ and $X$. So it holds

$$
R_{t}=Y_{t}^{\frac{1}{m+4}}, \quad U_{t}=k_{3}\left(1+X_{t}\right)^{2} Y_{t}^{-\frac{m+2}{m+4}} i(t), \quad t \in \mathcal{I} .
$$

The Monte Carlo method can be applied by simulating paths of the OUP and computing paths of the functions $U$ and $R$ numerically. The red line in Figures 2(a) and 2(c) show the estimated mean function of $U$, which was gained by 1000 simulations of the OUP. The distance between the analytical mean function (3.6) and the estimated mean function gained by the Monte Carlo method in relation to the analytical mean function is always less than $2.6 \%$ in the considered interval (see figure $3)$.

Figures 2(b) and 2(d) show a single path of the arc voltage $U$. The graph shows a slight trembling, but not as strong as it can be observed in real world data. Figure 2 (b) shows a random time varying amplitude of the arc voltage, similar as it can be observed in reality (see, e.g. [4] or [6]).

\section{Series expansions of the pathwise solution}

In this section let the considered time interval be a bounded interval $\mathcal{I}=\left[t_{0}, T\right]$ with $0 \leq t_{0}<T$. According to the Karhunen-Loève theorem the Ornstein-Uhlenbeck process restricted to a bounded time interval can be expanded as

$$
X_{t}=\sum_{k \in \mathbb{N}} \sqrt{\lambda}_{k} \phi_{k}(t) \xi_{k}, \quad t \in \mathcal{I}
$$

where $\left(\xi_{k}\right)_{k \in \mathbb{N}}$ is a sequence of independent standard normally distributed random variables, $\left(\lambda_{k}\right)_{k \in \mathbb{N}}$ are the eigenvalues and $\left(\phi_{k}\right)_{k \in \mathbb{N}}$ are the associated eigenfunctions of the covariance operator of $X$ (cf. e.g. [7], chapter 11). The eigenvalues $\left(\lambda_{k}\right)_{k \in \mathbb{N}}$ are positive and converge to zero. 


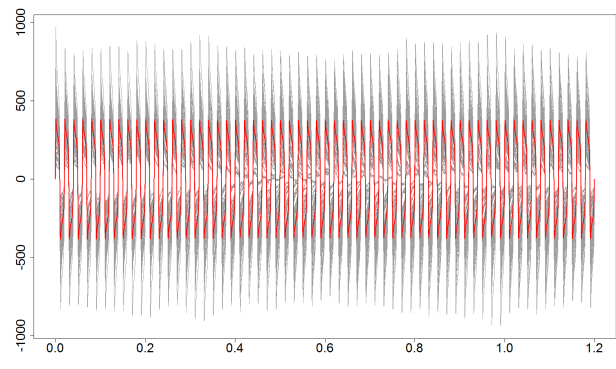

(a)

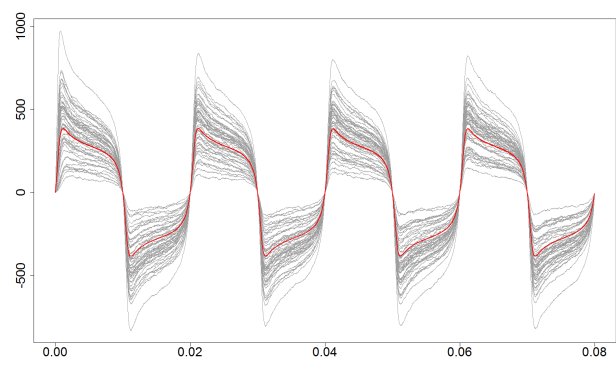

(c)

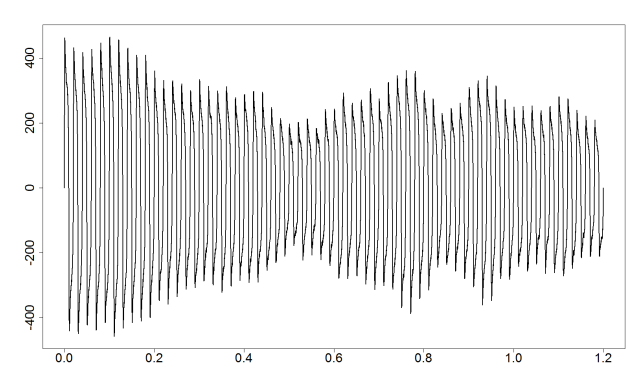

(b)

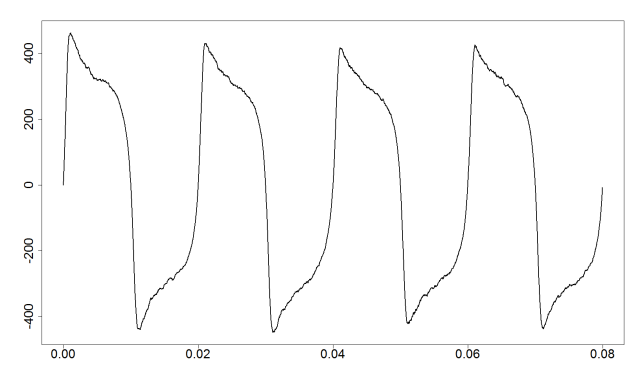

(d)

Figure 2. Left ( $a$ and $c$ ): estimated mean function of the arc voltage $U_{t}$ from 1000 simulated paths using Monte-Carlo method (red line) and 50 paths of $U_{t}$ (grey lines). Right ( $b$ and $d$ ): one single path of $U_{t}$ simulations using Monte Carlo method. The following parameters were used.

\begin{tabular}{cccccccccc}
\hline$a$ & $\omega$ & $m$ & $k_{1}$ & $k_{2}$ & $k_{3}$ & $\theta$ & $\sigma$ & $t_{0}$ & $y_{0}$ \\
\hline 75 & $100 \pi$ & 0 & 2000 & 5 & 35 & 0.5 & 0.5 & 0 & 6.58 \\
\hline
\end{tabular}

The series (4.1) converges in the function space $\mathrm{L}^{2}\left(\left[t_{0}, T\right]\right)$ as well as in the space $\mathrm{C}\left(\left[t_{0}, T\right]\right)$ almost surely and in the $p$-th mean for all $1 \leq p<\infty$ (cf. e.g. [2], chapter $3)$. For the OUP the eigenvalues can be calculated numerically and the eigenfunctions for given eigenvalues can be calculated analytically (cf. [3]).

The representation (4.1) can be applied to the random differential equation (3.2), which results in

$$
\frac{\mathrm{d} Y_{t}}{\mathrm{~d} t}=-\beta Y_{t}+f(t)\left(1+\sum_{k \in \mathbb{N}} \sqrt{\lambda}_{k} \phi_{k}(t) \xi_{k}\right)^{2}, \quad t \in \mathcal{I} .
$$

Expanding the square leads to an equation with a simple linear structure. This can be used to get a representation of the pathwise solution $Y_{t}$ in terms of the random variables $\left(\xi_{k}\right)_{k \in \mathbb{N}}$ and their products $\left(\xi_{k} \xi_{j}\right)_{k, j \in \mathbb{N}}$. 


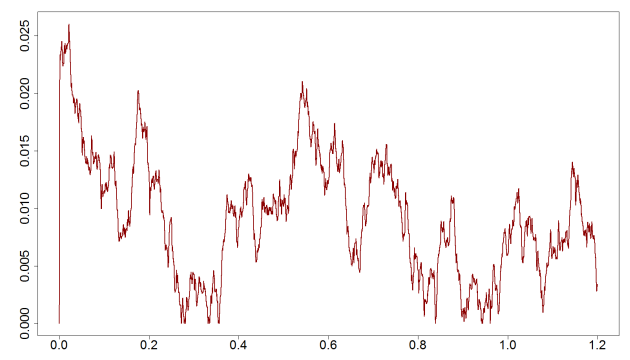

FIGURE 3. Graph of the function $t \mapsto \frac{\left|\mathrm{E}\left[Y_{t}\right]-\hat{\mathrm{E}}\left[Y_{t}\right]\right|}{\mathrm{E}\left[Y_{t}\right]}$, where $\hat{\mathrm{E}}\left[Y_{t}\right]$ is the estimated mean function estimated by the Monte Carlo method and $\mathrm{E}\left[Y_{t}\right]$ is the analytical mean function (3.6) with the same parameters as in figure 2.

Notice that these random variables do not form an orthogonal system. It holds

$$
Y_{t}=y_{0}(t)+\sum_{k \in \mathbb{N}} y_{k}(t) \xi_{k}+\sum_{k \in \mathbb{N}} y_{k k}(t) \xi_{k}^{2}+\sum_{\substack{k, j \in \mathbb{N}, k<j}} y_{k j}(t) \xi_{k} \xi_{j}, \quad t \in \mathcal{I}
$$

where the deterministic functions $y_{k}$ and $y_{k j}$ are solutions of deterministic linear ordinary differential equations with corresponding initial values. It holds

$$
\begin{array}{lrl}
\frac{\mathrm{d} y_{0}(t)}{\mathrm{d} t}=-\beta y_{0}(t)+f(t), & y_{0}\left(t_{0}\right)=y_{0}, \\
\frac{\mathrm{d} y_{k}(t)}{\mathrm{d} t}=-\beta y_{k}(t)+2 \sqrt{\lambda_{k}} \phi_{k}(t) f(t), & y_{k}\left(t_{0}\right)=0, \\
\frac{\mathrm{d} y_{k k}(t)}{\mathrm{d} t}=-\beta y_{k k}(t)+\lambda_{k} \phi_{k}^{2}(t) f(t), & y_{k k}\left(t_{0}\right)=0
\end{array}
$$

for $k \in \mathbb{N}$ and

$$
\frac{\mathrm{d} y_{k j}(t)}{\mathrm{d} t}=-\beta y_{k j}(t)+2 \sqrt{\lambda_{k} \lambda_{j}} \phi_{k}(t) \phi_{j}(t) f(t), \quad y_{k j}\left(t_{0}\right)=0
$$

for $k, j \in \mathbb{N}$ with $k<j$ and $t \in \mathcal{I}$. Explicit solutions can be given for these equations. It holds

$$
\begin{array}{lll}
y_{0}(t)=y_{0} e^{-\beta\left(t-t_{0}\right)}+\int_{t_{0}}^{t} f(s) e^{-\beta(t-s)} \mathrm{d} s, & t \in \mathcal{I}, \\
y_{k}(t)=2 \sqrt{\lambda_{k}} \int_{t_{0}}^{t} \phi_{k}(s) f(s) e^{-\beta(t-s)} \mathrm{d} s, & t \in \mathcal{I}, \\
y_{k k}(t)=\lambda_{k} \int_{t_{0}}^{t} \phi_{k}^{2}(s) f(s) e^{-\beta(t-s)} \mathrm{d} s, & t \in \mathcal{I}
\end{array}
$$

for $k \in \mathbb{N}$ and

$$
y_{k j}(t)=2 \sqrt{\lambda_{k} \lambda_{j}} \int_{t_{0}}^{t} \phi_{k}(s) \phi_{j}(s) f(s) e^{-\beta(t-s)} \mathrm{d} s, \quad t \in \mathcal{I},
$$


for $k, j \in \mathbb{N}$ with $k<j$. For numerical computations the sums in equation (4.3) have to be truncated, which gives an approximation of the solution $Y$. The representation (4.3) could be achieved due to the simple structure of the random differential equation $(3.2)$.

In general one can use an expansion of an already approximated solution in terms of orthogonal random variables, which is also known as polynomial chaos expansion. Therefore, let $Y^{N}$ denote the pathwise solution (3.3) of our initial value problem, where the $\operatorname{OUP}\left(X_{t}\right)_{t \in \mathcal{I}}$ is replaced by the truncated sum

$$
X_{t}^{N}:=\sum_{k=1}^{N} \sqrt{\lambda}_{k} \phi_{k}(t) \xi_{k}, \quad t \in \mathcal{I}
$$

with $N \in \mathbb{N}$. $\left(X_{t}^{N}\right)_{t \in \mathcal{I}}$ can be considered as a smoothed version of the OUP $\left(X_{t}\right)_{t \in \mathcal{I}}$. Then $Y^{N}$ can be represented as polynomial chaos expansion

$$
Y_{t}^{N}=\sum_{k=0}^{M} \tilde{y}_{k}(t) \Psi_{k}, \quad t \in \mathcal{I}
$$

with

$$
M=\frac{(N+2)(N+1)}{2}-1 \text {. }
$$

The $\left(\tilde{y}_{k}\right)_{0 \leq k \leq M}$ are deterministic functions and the $\left(\Psi_{k}\right)_{0 \leq k \leq M}$ are orthogonal random variables, for which applies that for every $\Psi_{k}$ exists a $N$-variate polynomial $p_{k}$, such that

$$
\Psi_{k}=p_{k}\left(\xi_{1}, \xi_{2}, \ldots, \xi_{N}\right),
$$

in particular $\Psi_{0}=1$ and $\Psi_{k}=\xi_{k}$ for $k \in\{1, \ldots, N\}$. In general the polynomial chaos expansion does not consist of a finite number of summands. But from the representation (4.3) follows, that one has to consider only polynomials up to degree two. It is convenient to choose the sequence $\left(\Psi_{k}\right)_{0 \leq k \leq M}$, such that the degrees of the associated polynomials $\left(p_{k}\right)_{0 \leq k \leq M}$ are increasing. In the considered case the sequence of polynomials can be determined by using the Hermite polynomials (cf. e.g. [7]). From the orthogonality of the random variables $\left(\Psi_{k}\right)_{0 \leq k \leq M}$ follows, that holds

$$
\mathrm{E}\left[Y^{N}(t) \Psi_{k}\right]=\tilde{y}_{k}(t) \cdot \mathrm{E}\left[\Psi_{k}^{2}\right]
$$

for all $k \in\{0,1, \ldots, M\}$ and $t \in\left[t_{0}, T\right]$. This results in representations of the coefficient functions $\left(\tilde{y}_{k}\right)_{0 \leq k \leq M}$. It holds

$$
\tilde{y}_{k}(t)= \begin{cases}y_{0} e^{-\beta\left(t-t_{0}\right)}+\int_{t_{0}}^{t} e^{-\beta(t-s)} f(s) \mathrm{d} s+\int_{t_{0}}^{t} e^{-\beta(t-s)} g_{0}(s) \mathrm{d} s, & \text { if } k=0 \\ \int_{t_{0}}^{t} e^{-\beta(t-s)} g_{k}(s) \mathrm{d} s, & \text { if } k \geq 1\end{cases}
$$

with $k \in\{0,1, \ldots, M\}$, where the functions $\left(g_{k}\right)_{0 \leq k \leq M}$ are defined by

$$
g_{k}(t):= \begin{cases}f(t) \sum_{j=1}^{N} x_{j}^{2}(t) & \text { if } k=0 \\ 2 f(t) x_{k}(t) & \text { if } 1 \leq k \leq N \\ f(t) \sum_{i=1}^{N} \sum_{j=1}^{N} x_{i}(t) x_{j}(t) M_{i j k} & \text { else }\end{cases}
$$


with

$$
x_{j}(t):=\sqrt{\lambda_{j}} \phi_{j}(t) \text { for } j \in\{1,2, \ldots, N\}
$$

and

$$
M_{i j k}:=\frac{\mathrm{E}\left[\Psi_{i} \Psi_{j} \Psi_{k}\right]}{\mathrm{E}\left[\Psi_{k}^{2}\right]} \text { for } i, j \in\{0,1, \ldots, M\}
$$

\section{Conclusion}

One modeling approach of the power system of electric arc furnaces leads to a nonlinear ordinary differential equation, which in some important cases can be solved with a linear differential equation for an auxiliary quantity. Real data show partly an irregular behaviour so that a stochastic modeling seems to be advisable.

In the present work one such stochastic model is investigated. Thereby one coefficient of the differential equation is replaced by a stochastic process, leading to a random differential equation and hence also to a stochastic voltage process. The input stochastic process is modelled with the help of a stationary Ornstein-Uhlenbeck process, for which many properties and results are known. The random differential equation is investigated with the help of Monte Carlo method, but also the usage of polynomial chaos expansions is explained.

The results show a relatively good agreement with real data. In the future we plan to investigate further methods and models and we also plan to investigate methods for statistical inference from real data for the considered models.

\section{References}

[1] Acha, E., Semlyen, A., Rajaković, N., A harmonic domain computational package for nonlinear problems and its application to electric arcs, IEEE Transactions on Power Delivery, 5(1990), no. 3, 1390-1397.

[2] Adler, R.J., Taylor, J.E., Random Fields and Geometry, Springer, New York, 2007.

[3] Corlay, S., Pagès, G., Functional quantization-based stratified sampling methods, Monte Carlo Methods Appl., De Gruyter, 21(2015), no. 1, 1-32.

[4] Grabowski, D., Selected Applications of stochastic approach in circuit theory, Wydawnictwo Politechniki Ślaskiej, Gliwice, 2015.

[5] Grabowski, D., Walczak, J., Analysis of deterministic model of electric arc furnace, 10th International Conference on Environment and Electrical Engineering, 1-4.

[6] Grabowski, D., Walczak, J., Klimas, M., Electric arc furnace power quality analysis based on stochastic arc model, 2018 IEEE International Conference on Environment and Electrical Engineering and 2018 IEEE Industrial and Commercial Power Systems Europe, 1-6.

[7] Sullivan, T.J., Introduction to Uncertainty Quantification, Springer, Cham, 2012. 
Hans-Jörg Starkloff

Technische Universität Bergakademie Freiberg

Faculty of Mathematics and Computer Science

Prüferstraße 9, 09599 Freiberg, Germany

e-mail: Hans-Joerg. Starklof $f @ m a t h . t u-f r e i b e r g . d e$

Markus Dietz

Technische Universität Bergakademie Freiberg

Faculty of Mathematics and Computer Science

Prüferstraße 9, 09599 Freiberg, Germany

e-mail: Markus.Dietz@math.tu-freiberg.de

Ganna Chekhanova

Technische Universität Bergakademie Freiberg

Faculty of Mathematics and Computer Science

Prüferstraße 9, 09599 Freiberg, Germany

e-mail: Anna.Chekhanova@math.tu-freiberg.de 